\title{
Accurate Superimposed Component Estimation for Improved Relay Performance during Power Swing
}

\author{
Subhadeep Paladhi, Ashok Kumar Pradhan, Senior Member, IEEE, and J. Ganeswara Rao
}

\begin{abstract}
There is continuous variation in amplitude, phase, and frequency of voltage and current signals during power swing in a power system. Such variation makes the memorized pre-fault data less accurate to be used as non-fault component while computing superimposed quantity for protection decisions during power swing. In this paper, the voltage and current signal characteristics during power swing are analyzed and a technique is proposed to obtain correct non-fault components for superimposed quantity computation. The accuracy of the method in estimating the non-fault components is observed for different power swing conditions simulated in a 39-bus New England system and field data. The relay performance improvement as observed is also provided while applying the method to directional relaying, fault type classification, and fault location applications for faults during power swing. Comparative assessment reveals the superiority of the proposed method in improving the accuracy of the superimposed components computed during power swing.
\end{abstract}

Index Terms-Power system faults, numerical relaying, superimposed component, power swing.

\section{INTRODUCTION}

\section{A. Motivation and Incitement}

$\mathbf{P}$ OWER swing is a rotor angle oscillation phenomena caused by the unbalance in generated and consumed power following disturbances like loss of generation, bulk load switching and line tripping [1], [2]. Such an oscillation results in continuous and significant variation in amplitude, phase, and frequency of voltage and current signals. The variation affects the performance of different protection schemes like distance relaying, directional relaying etc. [3]-[6]. Such degraded relay performance may result in maloperation at times and also may lead to cascading outages, as reported in [7], [8]. Severity and frequency of occurrence of power swing are in an increasing trend with the gradual reduction in power system inertia [9], [10]. Such a situation asks for improvement in the performance of network protection schemes to ensure reliable protection decisions during power swing.

This work was supported by the Central Power Research Institute, Bangalore, India under Grant RSOP/2019/TR/07.(Corresponding Author: Subhadeep Paladhi.)

Subhadeep Paladhi was with the Department of Electrical Engineering, Indian Institute of Technology, Kharagpur 721302, India. He is now with the Department of Electronic and Electrical Engineering, University of Strathclyde, G1 1XQ Glasgow, U.K. (e-mail: paladhisubha91@gmail.com).

Ashok Kumar Pradhan is with the Department of Electrical Engineering, Indian Institute of Technology, Kharagpur, India (email:akpradhan@ee.iitkgp.ac.in).

J. Ganeswara Rao is with Central Electricity Authority, India (e-mail: ganeshjada@gmail.com).

\section{B. Literature Review}

Modulation in voltage and current waveforms during power swing affects the performance of different network protection schemes for faults, occurred in such a situation [3], [11]. Zone-3 of distance relay backing up the adjacent power line covers a large operating region in impedance plane and is more vulnerable during power swing. Different methods are now being used to block the unintentional distance relay operation during power swing [12], [13]. Proper unblocking techniques are also used to ensure its correct operation for fault during swing. Unstable power swing may even lead to zone-1 malfunction at times. Supervisory methods are available in blocking its operation for such a situation [14], [15]. Fault type classification is a prerequisite to different relaying applications like distance relay and autoreclosing. Performance of sequence current based fault classification approaches is also found to be affected during power swing [16], [17]. Directional relay generally uses pre-fault voltage data for close-in fault situations. Large variation in voltage signal during power swing may lead such relays to malfunction at times [18]. Communication assisted protection schemes employing remote end signal (like current differential and synchrophasor based approaches) are immune to power swing but have issues associated with latency and communication failure [19], [20]. Superimposed component based approaches are being employed for different protection applications in both time and phasor domain, to reduce the impact of load variations and change in system conditions [21]-[24]. These methods compute superimposed components by using memorized pre-fault data as the non-fault component during fault for decision. Modulation of voltage and current waveforms during power swing changes their periodicity [25]. This results in erroneous computation of superimposed components, which may affect the performance of associated protection methods for fault during power swing [26]. So there is a scope to derive improved accuracy in computing the pure-fault component of voltage and current during power swing which is expected to enhance relay performance. Thereby a reliable protection scheme can be obtained.

\section{Contribution}

In this work, the performance of superimposed component based protection schemes is analyzed for fault during power swing and a method is proposed to compute superimposed components accurately improving their performance. The main contributions of this work are summarized below: 
1) Least square (LS) based technique is applied to demodulate the voltage and current signals during power swing using memorized pre-fault data and all the frequency components in the swing signals are extrapolated to obtain correct non-fault data in the fault period.

2) Superimposed components are computed by subtracting the extrapolated data (summation of all frequency components) from the measurements obtained during fault.

3) Each frequency component in the superimposed data is extracted by applying the LS based technique.

4) Protection decisions are derived in both phasor and time domain applications by applying any single frequency superimposed component therein, with proper frequency based adjustment in system impedances.

The performance of the proposition in acquiring non-fault component is tested for different power swing conditions simulated in 39-bus New England system using PSCAD/ EMTDC platform and field data obtained from Indian Power Grid. The compatibility of the proposed method is verified using OPAL-RT real-time simulation. The advantage of the proposed method while applying to different protection principles is demonstrated for fault during power swing. Comparative assessment reveals the superiority of the proposed method in superimposed component computation during power swing.

\section{Problem Statement}

This section demonstrates the limitation of the conventional approach in obtaining accurate superimposed component during power swing and evaluates its impact on the performance of relevant protection schemes.

\section{A. Challenges in superimposed component computation dur- ing power swing}

Available relays calculate superimposed components by subtracting few cycles (one or more) memorized data from the present measurements (voltage and current) with the time reference computed based on the steady state frequency. Voltage and current signals during power swing are modulated by low frequency components [25]. This affects the periodicity of the signals and results in a significant difference between the present and corresponding memorized data. This is demonstrated for a swing situation created in 39-bus New England system of Fig. 1 [27], [28]. A three-phase fault created in line 26-29 is cleared by opening the circuit breakers (CBs) at both ends of the line. As a result, a power swing is observed in the system. 2 shows the phase-A current in line $26-28$ seen by
Fig. 2 . the relay at bus 28 during the swing. Samples obtained from a 1-cycle data window (with a sampling rate of $1.2 \mathrm{kHz}$ ) are compared with the corresponding memorized data (2cycles earlier in this case) [24], [29]. A difference is observed between the present and memorized samples clearly, as well as the phasors estimated using both the data sets (shown in Fig. 2). With such a difference between present and memorized data, a significant superimposed component is obtained for a signal even without any fault in the system.

Such variation in signal results in erroneous computation of superimposed components for faults during power swing,

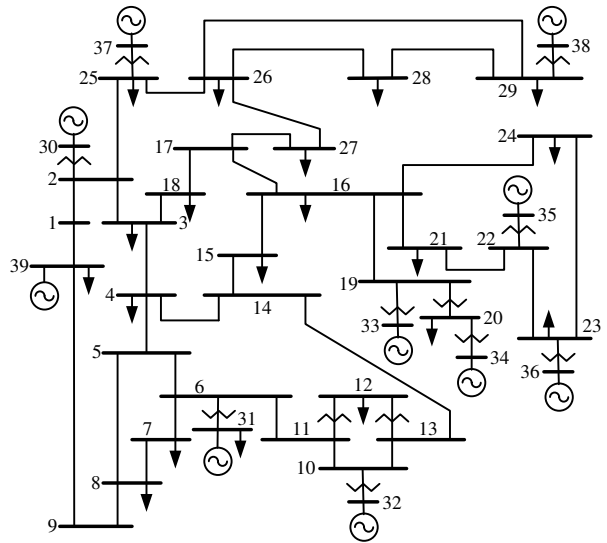

Fig. 1. 39-bus New England system.

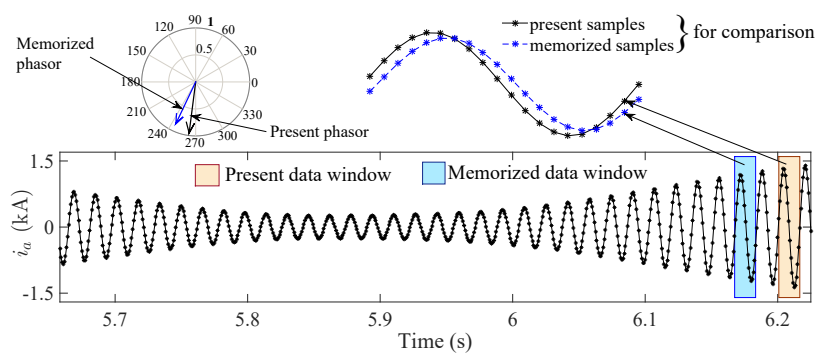

Fig. 2. Impact of current modulation on the signal periodicity.

when the memorized pre-fault data is used as the non-fault component during fault (a common practice employed in the available relays). In addition, such a conventional approach may even compute significant non-zero superimposed components for the healthy phases, which have an inevitable contribution in the calculation of sequence components. This is evident from the results shown in Fig. 3 for a phase-Ato-ground (AG) fault in line 26-28 of the system in Fig. 1, created at $6.3 \mathrm{~s}$ during the ongoing swing situation.
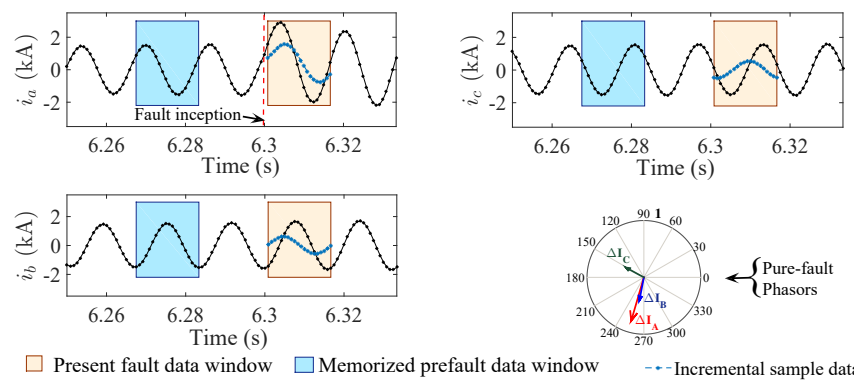

Fig. 3. Incremental phase currents for an AG fault during power swing.

\section{B. Performance of superimposed component based protection scheme during power swing}

With such erroneous computation of superimposed components for fault during power swing, performance of many available protection schemes may be affected. Such a case is demonstrated below with the performance of a superimposed positive sequence component based directional relay at bus 28 for the fault case mentioned above. Phase angle difference between positive sequence superimposed voltage and current 
components is used for identification of fault direction, as in (1) [30], [31]. $\Delta V_{1}$ and $\Delta I_{1}$ represent the positive sequence superimposed voltage and current components respectively. Such a technique is immune to the pre-fault loading conditions and also applicable to all fault types.

$$
\Phi=\angle \Delta V_{1}-\angle \Delta I_{1}=\left\{\begin{array}{l}
<0 ; \text { for forward fault } \\
>0 ; \text { for reverse fault }
\end{array}\right.
$$

Result in Fig. 4 shows that the directional relay at bus 28 identifies the forward AG fault in line 26-28 as a reverse fault. This shows the limited performance of the relay for a fault during power swing. Similar issues for relays can also be observed while performing other important functionalities (like fault type classification, fault distance calculation etc.) using superimposed component based approaches.

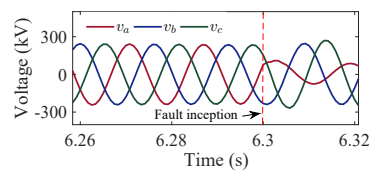

(a)

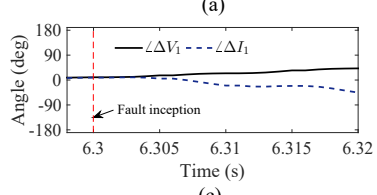

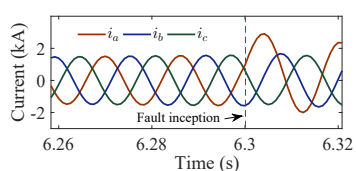

(b)

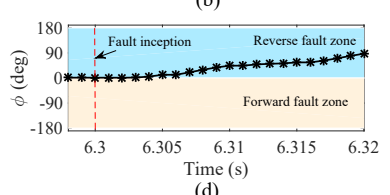

Fig. 4. Malfunction of directional relay during power swing showing (a) voltage, (b) current, (c) superimposed component angles and (d) relative angle.

Thus to improve the performance of such available protection schemes, there is a need of an alternative technique to calculate superimposed components correctly for faults during power swing.

\section{PROPOSED METHOD}

For a two bus equivalent power system during power swing, the voltage and current signals from both end sources contain two different frequency components [25], [32]. Thus the signals obtained by the relay can be modeled as,

$$
\begin{gathered}
v(t)=V_{m} \sin \left(2 \pi f_{m} t+\alpha_{m}\right)+V_{n} \sin \left(2 \pi f_{n} t+\alpha_{n}\right) \\
i(t)=I_{m} \sin \left(2 \pi f_{m} t+\beta_{m}\right)+I_{n} \sin \left(2 \pi f_{n} t+\beta_{n}\right)
\end{gathered}
$$

where $V_{m}$ and $V_{n}$ are the amplitudes of the voltage signal having frequencies $f_{m}$ and $f_{n}$, and the initial phase angles $\alpha_{m}$ and $\alpha_{n}$ respectively. Similarly, $I_{m}, I_{n}, \beta_{m}$ and $\beta_{n}$ represent the corresponding parameters of the current signal.

$$
\left[\begin{array}{c}
i\left(t_{0}\right) \\
i\left(t_{0}-\left(1 / f_{s}\right)\right) \\
\vdots \\
\vdots \\
i\left(t_{0}-(N-1) / f_{s}\right)
\end{array}\right]=\left[\begin{array}{cc}
-\sin (0) & \cos (0) \\
-\sin \left(2 \pi \frac{f_{m}}{f_{s}}\right) & \cos \left(2 \pi \frac{f_{m}}{f_{s}}\right) \\
\vdots & \vdots \\
\vdots & \vdots \\
-\sin \left(2 \pi \frac{(N-1) f_{m}}{f_{s}}\right) & \cos \left(2 \pi \frac{(N-1) f_{m}}{f_{s}}\right)
\end{array}\right.
$$

\section{can be expressed as in (6).}

$$
\left.\begin{array}{cc}
-\sin (0) & \cos (0) \\
-\sin \left(2 \pi \frac{f_{n}}{f_{s}}\right) & \cos \left(2 \pi \frac{f_{n}}{f_{s}}\right) \\
\vdots & \vdots \\
\vdots & \vdots \\
-\sin \left(2 \pi \frac{(N-1) f_{n}}{f_{s}}\right) & \cos \left(2 \pi \frac{(N-1) f_{n}}{f_{s}}\right)
\end{array}\right]\left[\begin{array}{c}
I_{m} \cos \beta_{m} \\
I_{m} \sin \beta_{m} \\
I_{n} \cos \beta_{n} \\
I_{n} \sin \beta_{n}
\end{array}\right]
$$

where $f_{s}$ is the sampling frequency, $\mathrm{N}$ is the number of samples per cycle and

$$
\begin{aligned}
& p_{1}=i_{k-2}, \quad p_{2}=\left(i_{k-3}+i_{k-1}\right) / 2 \\
& p_{3}=\left(i_{k-4}+2 i_{k-2}+i_{k}\right) / 4 \\
& q_{1}=i_{k-l-2}, \quad q_{2}=\left(i_{k-l-3}+i_{k-l-1}\right) / 2 \\
& q_{3}=\left(i_{k-l-4}+2 i_{k-l-2}+i_{k-l}\right) / 4
\end{aligned}
$$

$i_{k}$ represents the $k^{t h}$ sample of the current signal. $l=\frac{N}{4}$ is a suitable choice [25]. From (3), the frequencies of the current signal can be computed as in (4).

$$
f_{m}=\frac{\cos ^{-1}\left(\frac{D_{1}+\sqrt{D_{1}^{2}-4 D_{2}}}{2}\right) \cdot f_{s}}{2 \pi}
$$

where $D_{1}=\frac{\sum_{k=1}^{N}\left|p_{3} q_{1}-p_{1} q_{3}\right|_{k}}{\sum_{k=1}^{N}\left|p_{2} q_{1}-p_{1} q_{2}\right|_{k}}$ and $D_{2}=\frac{\sum_{k=1}^{N}\left|p_{3} q_{2}-p_{2} q_{3}\right|_{k}}{\sum_{k=1}^{N}\left|p_{2} q_{1}-p_{1} q_{2}\right|_{k}}$.

In order to extract the individual frequency component from the signal, the current signal in (2) is expanded as in (5).

$$
\begin{aligned}
i(t) & =I_{m} \cos \beta_{m} \sin \left(2 \pi f_{m} t\right)+I_{m} \sin \beta_{m} \cos \left(2 \pi f_{m} t\right) \\
& +I_{n} \cos \beta_{n} \sin \left(2 \pi f_{n} t\right)+I_{n} \sin \beta_{n} \cos \left(2 \pi f_{n} t\right)
\end{aligned}
$$

Using one cycle current samples during prefault, the frequency information obtained from (4) and $t_{0}$ as the reference time, (5)$$
\left.f_{s}\right)
$$

The unknown matrix $\left[X_{i}\right]$ can be estimated using Least-square technique as in (8). In this way, the current amplitudes $\left(I_{m}\right.$ and

$$
\left[M_{i}\right]=[A]\left[X_{i}\right] .
$$




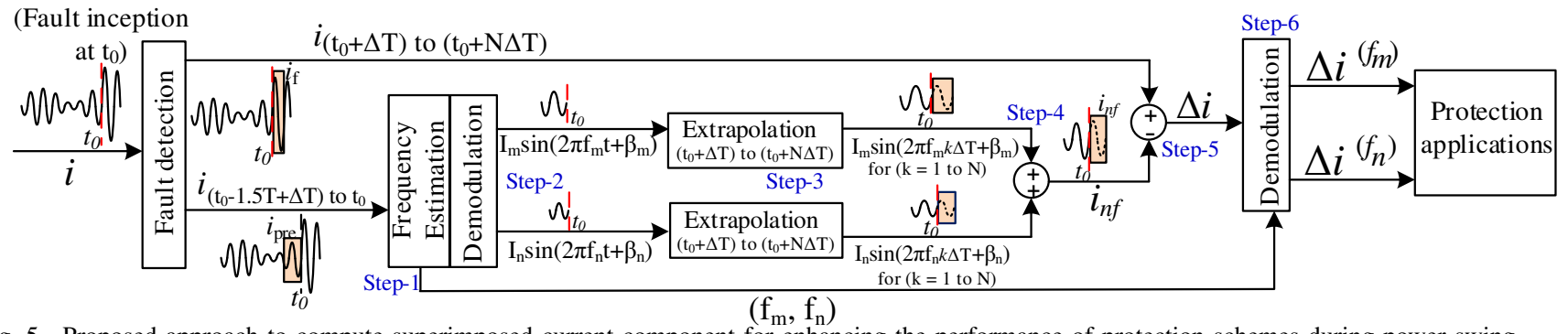

Fig. 5. Proposed approach to compute superimposed current component for enhancing the performance of protection schemes during power swing.

$\left.I_{n}\right)$ and phase angles $\left(\beta_{m}\right.$ and $\left.\beta_{n}\right)$ at $t_{0}$ can be determined.

$$
\left[X_{i}\right]=[A]^{+}\left[M_{i}\right]
$$

where $[A]^{+}=\left[[A]^{T}[A]\right]^{-1^{2}}[A]^{T}$

In a similar way, $V_{m}, V_{n}, \alpha_{m}$ and $\alpha_{n}$ associated with the voltage signals in (2) can also be estimated.

\section{B. Computation of superimposed components for faults during} power swing

A protection scheme using superimposed component, calculated by subtracting one or two cycles earlier memorized prefault data from present measurement, should take a decision within a cycle following fault inception [33]. Non-fault sample corresponding to each frequency component of the swing current signal in (2) can be predicted using (9), with the frequency, amplitude, and phase information estimated using (4) and (6). Consider $t_{0}$ in (6) as the fault inception instant.

$$
\begin{aligned}
i_{t f_{k}}^{\left(f_{m}\right)} & =I_{m} \sin \left(2 \pi f_{m} k \Delta T+\beta_{m}\right) \\
i_{t f_{k}}^{\left(f_{n}\right)} & =I_{n} \sin \left(2 \pi f_{n} k \Delta T+\beta_{n}\right)
\end{aligned}
$$

where $i_{t f_{k}}$ is the $k^{t h}$ sample of the current signal following fault inception and $\Delta T$ is the time interval between two consecutive samples. Superscripts represent the frequency of corresponding signal. With $\mathrm{k}$ value of 1 to $\mathrm{N}$, each of the signals in (9) can be extrapolated for 1-cycle following fault inception. Thus the non-fault component for the period can be obtained as,

$$
i_{n f}=i_{t f}^{\left(f_{m}\right)}+i_{t f}^{\left(f_{n}\right)} .
$$

By subtracting the non-fault component obtained using (10) from the present measurement, the correct superimposed current can be calculated. This is also comprised of two frequency components and can be expressed as,

$$
\begin{aligned}
\Delta i(t)= & \Delta I_{m} \sin \left(2 \pi f_{m} k \Delta T+\gamma_{m}\right) \\
& +\Delta I_{n} \sin \left(2 \pi f_{n} k \Delta T+\gamma_{n}\right)+I_{0} e^{\frac{-k \Delta T}{\tau}}
\end{aligned}
$$

where $\Delta I_{m}$ and $\Delta I_{n}$ are the amplitudes of the superimposed current signal having frequencies $f_{m}$ and $f_{n}$, and $\gamma_{m}$ and $\gamma_{n}$ are the phase angles at fault inception instant. $I_{0} e^{\frac{-k \Delta T}{\tau}}$ is the decaying-DC component observed following fault inception. In a similar way, the superimposed voltage is expressed as,

$$
\Delta v(t)=\Delta V_{m} \sin \left(2 \pi f_{m} k \Delta T+\theta_{m}\right)+\Delta V_{n} \sin \left(2 \pi f_{n} k \Delta T+\theta_{n}\right)
$$

The equivalent reactance of the network varies with changes in system frequency. Thus the performances of the protection schemes, which decide based on system impedance information, may be affected during power swing. As an example, a superimposed component based directional relay calculates the phase angle of equivalent source impedance for its decision. Therefore the frequency components of the superimposed signals, in (11) and (12), are to be demodulated using leastsquare technique, similar to (6) and required to be adjusted to fundamental frequency component properly before applying to the protection applications. The decaying-DC component in (11) can be eliminated in least-square estimation by modeling it using Taylor series expansion [34].

\section{Proposed approach}

The proposed method is to be initiated when the power swing condition is detected in a power system [1], [25]. During no power swing condition, $f_{m}$ and $f_{n}$ are almost equal, and the value of $\sum_{k=1}^{N}\left|p_{2} q_{1}-p_{1} q_{2}\right|_{k}$ becomes close to zero for a long period of time. Therefore, the proposed method based on double-frequency signals cannot be applied. The nonswing condition can be detected by setting a threshold $(\zeta)$ to $\sum_{k=1}^{N}\left|p_{2} q_{1}-p_{1} q_{2}\right|_{k}$. A suitable value of $\zeta$ is considered as $40 \%$ of the full-load current [25]. The steps followed in the proposed approach to enhance the performance of superimposed component based protection scheme during power swing are provided below, which are also shown in Fig. 5 for only current signal. Similar steps are followed for voltage signals.

Step 1: Extract the multiple frequency information of a swing signal using (4), with approximately one-and-a-half cycle samples during pre-fault.

Step 2: Demodulate the swing signal by computing the amplitudes and phases of both frequency components using least-square technique, as in (6).

Step 3: Extrapolate each frequency component for 1-cycle following fault inception using (9).

Step 4: Calculate the non-fault component using (10), with the sample values extrapolated for each frequency component in Step 3.

Step 5: Compute the superimposed component by subtracting the non-fault samples (obtained in Step 4) from the present measurement.

Step 6: Demodulate the frequency components of the superimposed signals in (11) and (12) using least-square based technique and apply in the protection schemes, as required.

\section{Computational requirement}

One-cycle summation of both the equations in (3) are obtained using the moving window. The frequency estimation 
using (4) requires six real multiplications, two real divisions, nine real summations, and the calculation of the solution to one quadratic equation. Each phasor computation using least square technique in (6), with a window length of $\mathrm{N}$ samples requires $4 N$ real multiplications and $4(N-1)+2$ real summations. In order to reduce the computational burden, the pseudoinverse in (8) for the required frequency combination is calculated offline and stored in memory [35]. The computational burden can be further minimized by keeping the sampling rate low. In terms of memory, the method needs to store approximately one-and-a-half-cycle sample values. This requires additional memory of approximately $9 \mathrm{MB}$ $(4 \times 7 \times 4 \times 2 \times 200 \times 200$ B $)$ to store the elements of the first four rows of the pseudoinverse matrix in (8). Up to fifth harmonic, and decaying dc are considered. The higher order harmonics are removed from the signals using the antialiasing filter. The computational burden and memory requirement is found to be compatible with the present technology being used for relay applications.

\section{REsults}

Performance of the proposed extrapolation technique for estimating the non-fault component during power swing is tested for different power swing situations created in 39-bus New England system using PSCAD simulation platform. The performance of the proposed method is also tested with field data obtained from the Indian Power Grid. The advantage of the proposed method in improving relay performance is demonstrated by applying it to three protection applications. The swing situations are created as mentioned in Section-II.

\section{A. Verifying proposed extrapolation approach during swing}

1) With variation in swing frequency: The proposed method demodulates the swing signal during pre-fault and then extrapolates the individual frequency component to obtain correct non-fault quantity for the situation. The performance of such an approach is tested for different swing signals created in the 39-bus system of Fig. 1. The non-fault swing signals are compared with the extrapolated signals to verify the tracing capability of the technique. Starting time for extrapolation $\left(t_{0}\right)$ is varied to test the performance of the approach in acquiring non-fault signals for different fault inception angles. Results in Fig. 6 demonstrate the performance of relay at bus 28 in extrapolating phase-A current signals in line 26-28 during power swing, with swing frequencies $\left(f_{s w}\right)$ of $1 \mathrm{~Hz}, 4 \mathrm{~Hz}$, $7 \mathrm{~Hz}$, and $10 \mathrm{~Hz}$ respectively. Corresponding power angles $(\delta)$ set for all the four cases are $20^{\circ}, 60^{\circ}, 120^{\circ}$, and $170^{\circ}$. Results show that the extrapolated signals follow the actual measurements for all the cases. This demonstrates the accurate performance of the proposed method in estimating correct nonfault component during power swing. The adaptivity with high swing frequency advocates the applicability of the proposed approach even for a low inertia system.

Note: The extrapolation performed for several cycles is to demonstrate the accuracy of the proposed technique in nonfault swing situations, whereas it is required to be performed only for a cycle following fault inception to apply in protection applications.

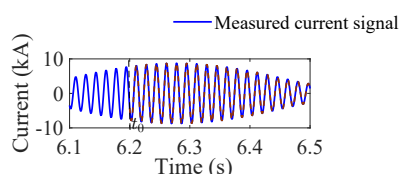

(a)

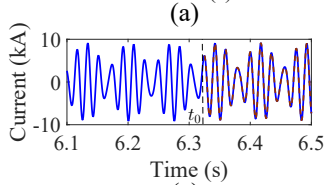

(c)

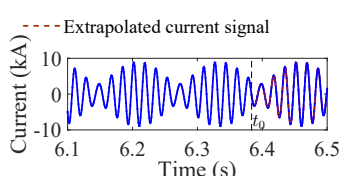

(b)

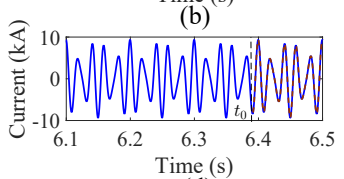

(d)
Fig. 6. Performance of proposed extrapolation technique for swing signals with (a) $f_{s w}=1 \mathrm{~Hz}, \delta=20^{0}, t_{0}=6.20 \mathrm{~s}$, (b) $f_{s w}=4 \mathrm{~Hz}, \delta=60^{\circ}, t_{0}=$ $6.38 \mathrm{~s}$, (c) $f_{s w}=7 \mathrm{~Hz}, \delta=120^{\circ}, t_{0}=6.33 \mathrm{~s}$ and (d) $f_{s w}=10 \mathrm{~Hz}, \delta=$ $170^{0}, t_{0}=6.38 \mathrm{~s}$.

2) In the presence of measurement noise: One-cycle summation of numerators and denominators associated with $D_{1}$ and $D_{2}$ in (3) weakens the effect of noise in frequency estimation. In order to test the performance of the proposed extrapolation technique in the presence of noise, a test current signal is generated as in (2) with $I_{m}=200 A, I_{n}=$ $150 \mathrm{~A}, f_{m}=58.5 \mathrm{~Hz}, f_{n}=61.5 \mathrm{~Hz}, \beta_{m}=\frac{\pi}{4}$ and $\beta_{n}=-\frac{\pi}{6}$. The signal is contaminated with uniform distribution noise with zero mean and a standard deviation of $0.5 \%$. Results shown in Fig. 7(a) demonstrate that the proposed method can correctly estimate the frequencies during power swing even in the presence of noise. Extrapolated signal in Fig. 7(b) also follows the actual signal.

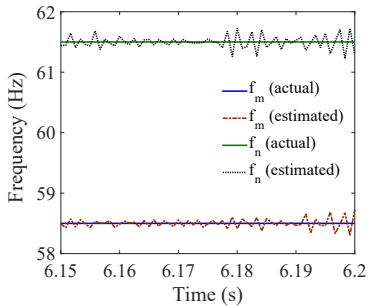

(a)

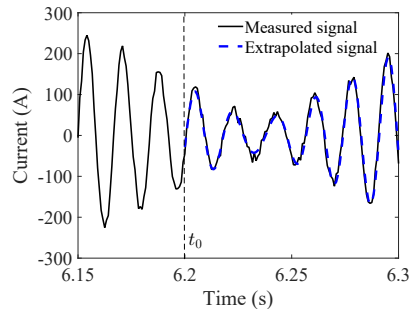

(b)
Fig. 7. (a) Estimated frequencies and (b) extrapolated signal generated in the presence of noise.

3) In the presence of harmonics: In order to test the performance of the proposed extrapolation technique in the presence of harmonics, all the lower order harmonics $\left(\leq 5^{\text {th }}\right.$ order) are added in the same test signal considered in section IV.A.(2), as per IEEE Std. 519-1992. All the higher order harmonics are filtered out using anti-aliasing filter. Results shown in Fig. 8(a) demonstrate that the estimated frequencies are very close to the actual frequencies and the extrapolated signal in Fig. 8(b) closely follows the actual signal for the first 2 cycles after $t_{0}$. The small deviation can also be eliminated with improved filtering technique.

4) With varying swing frequency: The swing frequency may not remain constant with time. It changes very slowly in a power system. In order to verify the performance of the proposed method in such a situation, a test signal is generated as in (13).

$$
i(t)=I_{m} \sin \left(2 \pi(f-\Delta f) t+\beta_{m}\right)+I_{n} \sin \left(2 \pi(f+\Delta f) t+\beta_{n}\right)
$$




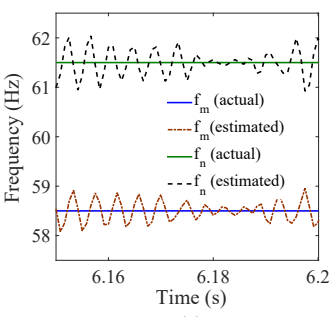

(a)

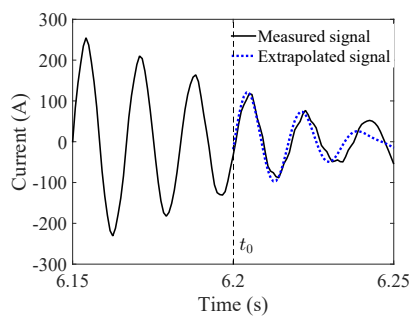

(b)
Fig. 8. (a) Estimated frequencies and (b) extrapolated signal generated in the presence of harmonics.

where $I_{m}=1.5 \mathrm{kA}, I_{n}=1 \mathrm{kA}, f=60 \mathrm{~Hz}, \beta_{m}=\frac{\pi}{6}$ and $\beta_{n}=-\frac{\pi}{4} . \Delta f$ has an initial value of $0.5 \mathrm{~Hz}$ and changes at a rate of $1.5 \mathrm{~Hz} / \mathrm{s}$ and $2.5 \mathrm{~Hz} / \mathrm{s}$ for two cases respectively. The signal is sampled with a rate of $1.2 \mathrm{kHz}$. Results shown in Fig. 9 demonstrate that the extrapolated signals closely follow the actual signal even for such situations. The extrapolation is performed at $0.64 \mathrm{~s}$ for both the cases.

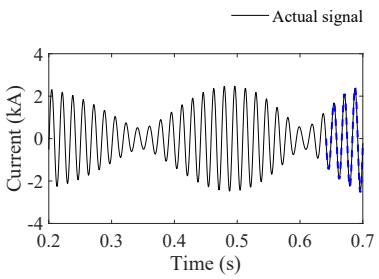

(a)

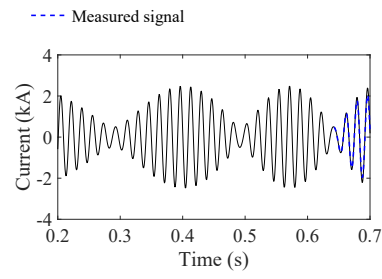

(b)
Fig. 9. Performance of the proposed method with change in swing frequency at a rate of (a) $1.5 \mathrm{~Hz} / \mathrm{s}$ and (b) $2.5 \mathrm{~Hz} / \mathrm{s}$.

5) For a three machine equivalent system: A multi-machine system may consist of more than two coherent areas, where frequency of each area may deviate from nominal frequency. In order to verify the performance of the proposed method for such a situation, a test current signal is generated as in (14), considering a three machine equivalent system.

$$
\begin{aligned}
i(t)= & I_{1} \sin \left(2 \pi f_{1} t+\beta_{1}\right)+I_{2} \sin \left(2 \pi f_{2} t+\beta_{2}\right) \\
& +I_{3} \sin \left(2 \pi f_{3} t+\beta_{3}\right)
\end{aligned}
$$

where, $I_{1}=2 k A, I_{2}=1.5 k A, I_{3}=1 k A, f_{1}=58 \mathrm{~Hz}, f_{2}=$ $61 \mathrm{~Hz}, f_{3}=59 \mathrm{~Hz}, \beta_{1}=\frac{\pi}{4}, \beta_{2}=-\frac{\pi}{6}$ and $\beta_{1}=\frac{\pi}{18}$ Results in Fig. 10(a) shows that the estimated frequencies are not constant for three machine equivalent system, but not changes rapidly with time. Fig. 10(b) demonstrates that the extrapolated signal generated by the proposed method follows the actual signal correctly even for such a situation. This justifies the signal model considered in (2) to be proper for this work.

6) With field data during power swing: In order to validate the accuracy of the proposed method with field data, the method is applied to a sampled current data of a power swing condition collected from the Indian Power Grid $(50 \mathrm{~Hz}$ system). Result is shown in Fig. 11. The extrapolated signal generated by the proposed method is observed to overlap with the actual measurement signal. This validates the accuracy of the proposed method while applied to field data. (a)

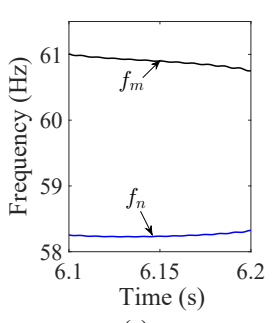

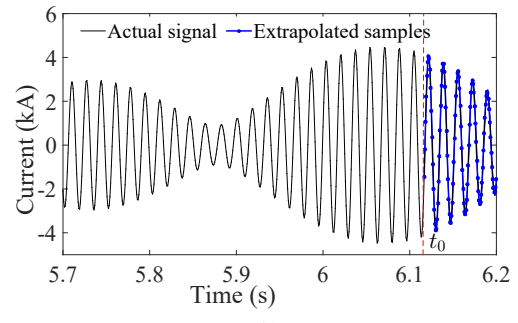

(b)
Fig. 10. Performance of the proposed method with three-machine equivalent system.

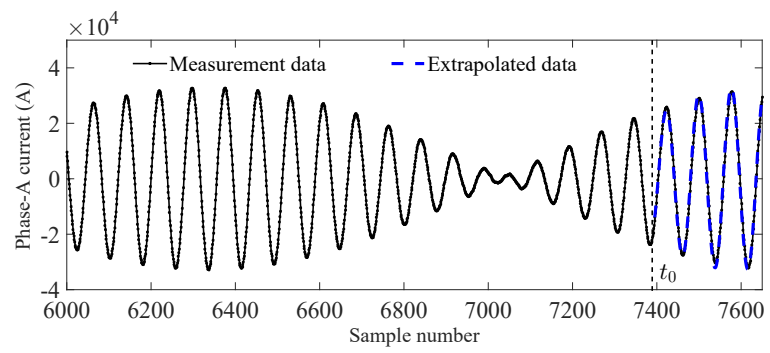

Fig. 11. Performance of the proposed method with field data.

\section{B. Performance of the proposed method for fault type classi- fication during power swing}

Fault type classification is an integral part of relay decision process for transmission networks required for different applications such as in single pole tripping, distance relaying, etc. For fault type classification, phase angle comparison of the superimposed sequence currents is a common technique, as shown in Fig. 12 [36], [37].

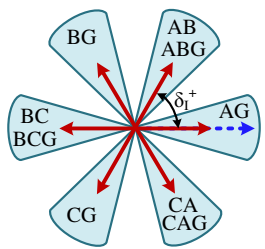

(a) $\delta_{I}^{+}$comparator

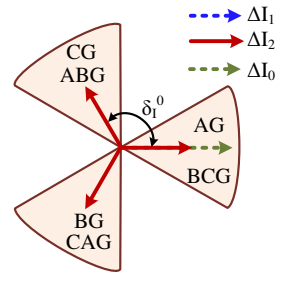

(b) $\delta_{\mathrm{I}}^{0}$ comparator
Fig. 12. Current angle-based fault type classifying logic, (a) $\delta_{I}^{+}$comparator and (b) $\delta_{I}^{0}$ comparator.

In the figure, $\mathrm{A}, \mathrm{B}, \mathrm{C}$, and $\mathrm{G}$ represent three phases and ground respectively and subscripts 0,1 , and 2 are for sequence components. $\delta_{I}^{+}$and $\delta_{I}^{0}$ are the angle of negative sequence superimposed current relative to the positive and zero sequence superimposed currents respectively. Inaccuracy in obtaining correct superimposed current components during power swing may result in misidentification of fault type with such an approach. This is demonstrated for a phase-to-phase fault (AB type), created in line 26-28 at a distance of $0.4 p u$ from bus 28 with a fault resistance $\left(R_{F}\right)$ of $2 \Omega$ during a power swing situation. The current signals for the situation is shown in Fig. 13 (a). Presence of multiple frequency components modulates the amplitude and phase angles of the fundamental signal and the conventional approach of using 2cycle memorized data as the non-fault component computes 


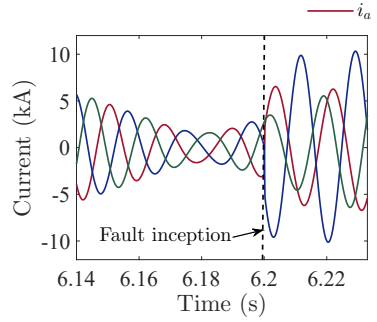

(a)

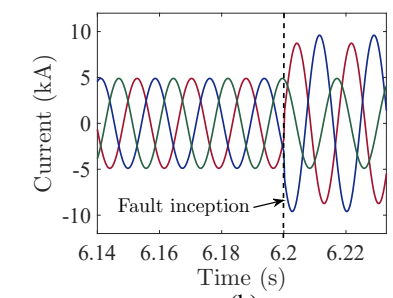

(b)
Fig. 13. (a) Current measured by the relay for an $\mathrm{AB}$ fault during power swing, (b) single frequency component of the measured current signal.

the superimposed phase currents incorrectly. The performance of fault type classifier with such improper superimposed component is demonstrated in Fig. 14. Result shows that the fault classifier using conventional approach identifies the fault as BG type using $\delta_{I}^{+}$comparator of Fig. 12 instead of AB. On the other hand, the proposed method extracts a single frequency component accurately by demodulating the swing current signal and computes the correct superimposed component of the corresponding frequency. The swing frequency observed at bus 28 is $3 \mathrm{~Hz}$. The current signal with a single frequency component $(57 \mathrm{~Hz})$ extracted from the actual measured signal is shown in Fig. 13 (b). Result in Fig. 14 demonstrates that the fault classifier identifies the fault type correctly using proposed approach.

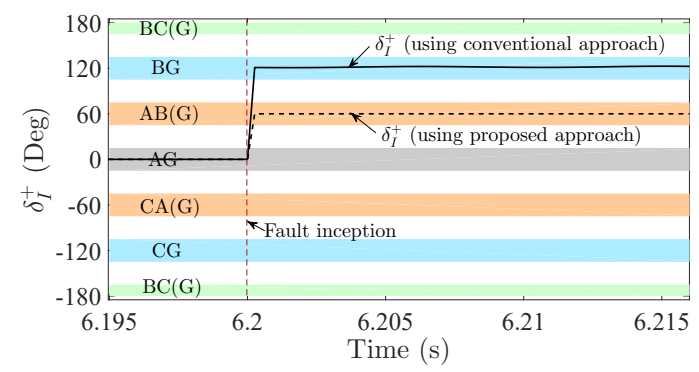

Fig. 14. Performance of the proposed fault classification method for AB fault.

With homogeneity of the conventional transmission network, the phase angle of the superimposed current component measured by the relay is the same as that of currents in the faulted path. This is valid for each single frequency component in a power network. Thus the performance of the current angle based classifier applying the proposed approach remains unaffected by $R_{F}$. Performances of fault classifier applying both conventional and proposed approaches are provided in Table I for variation in $\mathrm{R}_{\mathrm{F}}$. Results show that $\delta_{I}^{+}$and $\delta_{I}^{0}$ calculated by the proposed method are within the correct classification zones as mentioned in column II of Table I, whereas the conventional approach fails to identify the fault types correctly for some cases. For the case of BCG fault with $R_{F}=100 \Omega$ (Second row of Table I), the conventional approach calculates $\delta_{I}^{+}=71.88^{0}$, which lie outside the classifier zone setting of BCG fault $\left(180^{0} \pm 15^{0}\right)$. On the other hand, the relay using proposed method calculates $\delta_{I}^{+}=-173.56^{0}$ and $\delta_{I}^{0}=-9.23^{0}$ and identifies the fault type correctly. This clearly demonstrates the superiority of the proposed approach in enhancing the performance of the current angle based fault classifier during power swing.

TABLE I

PERFORMANCE OF FAULT CLASSIFIER FOR VARIATION IN $\mathrm{R}_{\mathrm{F}}$

\begin{tabular}{|c|c|c|c|c|c|c|}
\hline \multirow{2}{*}{$\begin{array}{l}\text { Fault } \\
\text { Type }\end{array}$} & \multirow{2}{*}{$\begin{array}{l}\text { Classifier Zone } \\
\text { Setting (Fig. 12) }\end{array}$} & \multirow{2}{*}{$\begin{array}{l}R_{F} \\
(\Omega)\end{array}$} & \multicolumn{2}{|c|}{ Conventional } & \multicolumn{2}{|c|}{ Proposed } \\
\hline & & & $\delta_{I}^{+}$ & $\delta_{I}^{0}$ & $\delta_{I}^{+}$ & $\delta_{I}^{0}$ \\
\hline \multirow{2}{*}{ AG } & \multirow{2}{*}{$\begin{array}{l}\delta_{I}^{+}: 0 \pm 15 \\
\delta_{I}^{0}: 0 \pm 30\end{array}$} & 5 & -99.87 & 0.88 & 0.00 & -0.17 \\
\hline & & 100 & -101.51 & 1.34 & 0.00 & -0.17 \\
\hline \multirow[t]{2}{*}{$\mathrm{BCG}$} & \multirow{2}{*}{$\begin{array}{l}\delta_{I}^{+}: 180 \pm 15 \\
\delta_{I}^{0}: 0 \pm 30\end{array}$} & 5 & 74.24 & -12.68 & 177.52 & -4.74 \\
\hline & & 100 & 71.88 & -10.28 & -173.36 & -9.23 \\
\hline $\mathrm{CA}$ & $\delta_{I}^{+}:-60 \pm 15$ & 1 & -162.54 & - & -60.00 & - \\
\hline
\end{tabular}

\section{Performance of the proposed method for fault direction identification during power swing}

Superimposed component based directional relaying is accomplished in both time and phasor domain applications. Performance of such relaying schemes is tested in this section for faults during power swing.

1) Performance of phasor based superimposed component direction relaying during power swing: A directional relay uses phase angle difference between positive sequence superimposed voltage and current phasors, as in (1) for acquiring decision [30], [31]. Incorrect computation of superimposed components during power swing may result in malfunction of such relays at times. One such malfunction scenario is demonstrated in Section-II.B. In this section, the performance of the proposed method is demonstrated while preventing such malfunctioning of directional relays.

The proposed method demodulates the superimposed voltage and current signals and extracts the frequency components. Ratio of superimposed voltage and current of any single frequency provides the negative of equivalent source impedance ( $Z_{s}=R_{s}+j X_{s}$ ) corresponding to the relay. This impedance angle is used by the relay for acquiring decision. As the reactance $\left(X_{s}\right)$ is dependent on system frequency, it should be adjusted, as in (15), for the correct decision.

$$
\begin{aligned}
\Phi & =\tan ^{-1}\left(\frac{\operatorname{Im}\left(\frac{\Delta V^{\left(f_{m}\right)}}{\Delta I^{(f m)}}\right) \cdot\left(\frac{f}{f_{m}}\right)}{\operatorname{Re}\left(\frac{\Delta V^{\left(f_{m}\right)}}{\Delta I^{\left(f_{m}\right)}}\right)}\right) \\
& =\left\{\begin{array}{l}
<0 ; \text { for forward fault } \\
>0 ; \text { for reverse fault }
\end{array}\right.
\end{aligned}
$$

Result in Fig. 15 (a) shows the performance of directional relay at bus 28 using proposed and convention approaches for a forward three-phase fault created in line 26-28. The result demonstrates that the relay using the proposed approach performs correctly for the situation, where the conventional approach identifies the fault in the reverse direction. Fig. 15 (b) and (c) show the positions of superimposed voltage and current components computed by the relay after 1-cycle following fault inception by applying conventional and proposed approaches respectively.

The angle $\phi$ calculated by the directional relay at bus 28 using (1) and (15), applying conventional and proposed 
approaches is provided in Table II demonstrating the performance for different fault types created in line 26-28 and line 28-29 with change in fault resistance and swing frequency. Results show that the proposed method calculates $\phi$ as negative for forward faults and positive for reverse faults and identifies the fault direction correctly for all the cases. On the other hand, the conventional approach fails to identify the fault direction correctly for some cases. The shaded boxes in the Table show the maloperation cases using the conventional approach. Thus the superiority of the proposed method is confirmed in enhancing the performance of directional relay in phasor domain.

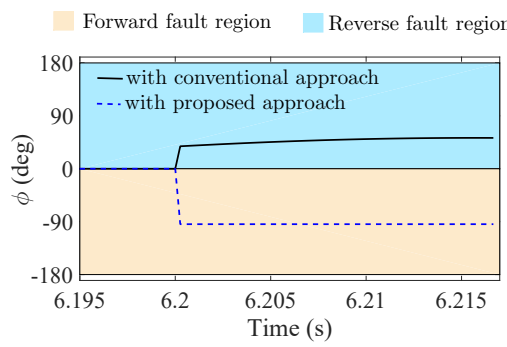

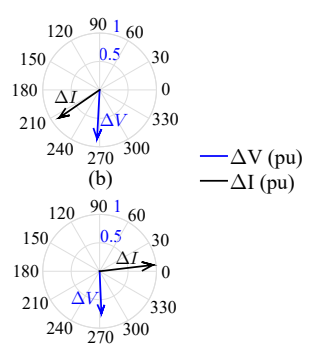

Fig. 15. (a) Performance of superimposed component based phasor-domain directional relaying with superimposed voltage and current phasors computed using (b) conventional and (c) proposed approach.

TABLE II

PERFORMANCE OF DIRECTIONAL RELAY FOR DIFFERENT FAULT TYPES With CHANGE IN FAUlt RESISTANCE and SWING FREQUENCY

\begin{tabular}{|c|c|c|c|c|c|c|}
\hline \multirow{2}{*}{$\begin{array}{l}\text { Fault } \\
\text { Type }\end{array}$} & \multirow{2}{*}{$\begin{array}{l}f_{s w} \\
(\mathrm{~Hz})\end{array}$} & \multirow{2}{*}{$\begin{array}{l}R_{F} \\
(\Omega)\end{array}$} & \multicolumn{2}{|c|}{$\begin{array}{c}\phi_{\text {forward }}(\mathrm{deg}) \\
\text { (for fault in line 26-28) }\end{array}$} & \multicolumn{2}{|c|}{$\begin{array}{c}\phi_{\text {reverse }}(\mathrm{deg}) \\
\text { (for fault in line 28-29) }\end{array}$} \\
\hline & & & Conventional & Proposed & Conventional & Proposed \\
\hline \multirow{4}{*}{ AG } & \multirow{2}{*}{1} & 5 & -8.75 & -94.99 & 171.25 & 85.23 \\
\hline & & 100 & 33.36 & -94.98 & -146.64 & 85.23 \\
\hline & \multirow{2}{*}{5} & 5 & 90.74 & -94.90 & -110.71 & 85.23 \\
\hline & & 100 & 106.97 & -94.94 & -96.88 & 85.23 \\
\hline \multirow{4}{*}{ BCG } & \multirow{2}{*}{1} & 1 & -31.14 & -94.99 & 138.77 & 85.00 \\
\hline & & 50 & -26.56 & -94.99 & 142.34 & 85.01 \\
\hline & \multirow{2}{*}{5} & 1 & 56.16 & -94.99 & -133.81 & 85.01 \\
\hline & & 50 & 60.09 & -94.99 & -133.01 & 85.01 \\
\hline \multirow{2}{*}{$\mathrm{AB}$} & 1 & 1 & -35.44 & -94.99 & 144.56 & 85.01 \\
\hline & 5 & 1 & 47.20 & -94.99 & -132.80 & 85.01 \\
\hline \multirow{2}{*}{$\mathrm{ABC}$} & 1 & 1 & -59.63 & -94.96 & 87.96 & 85.21 \\
\hline & 5 & 1 & 8.71 & -94.97 & 62.48 & 85.21 \\
\hline
\end{tabular}

2) Performance of time-domain superimposed component direction relaying: In order to acquire faster decisions by breaking the limitation of phasor computation, directional relaying can be accomplished in time-domain. Such an approach is presented in [21].

For an AG fault in forward direction of a two bus equivalent system, the voltage drop relation across source side can be expressed as [21],

$$
\Delta v_{a}=-\left|Z_{s}\right|\left(\frac{R_{s}}{\left|Z_{s}\right|} \cdot \Delta i_{a}+\frac{L_{s}}{\left|Z_{s}\right|} \cdot \frac{\mathrm{d} \Delta i_{a}}{\mathrm{~d} t}\right)
$$

where $Z_{s}=R_{s}+j\left(2 \pi f L_{s}\right)$ represents the equivalent impedance of the source side at relay bus.
The relation in (16) can be rewritten as,

$$
\Delta v_{a}=-\left|Z_{s}\right| \cdot \Delta i_{a z}
$$

where $\Delta i_{a z}=\left(\frac{R_{s}}{\left|Z_{s}\right|} \cdot \Delta i_{a}+\frac{L_{s}}{\left|Z_{s}\right|} \cdot \frac{\mathrm{d} \Delta i_{a}}{\mathrm{~d} t}\right)$ represents the replica current waveform of phase-A. Thus the sample wise multiplication of $\Delta v_{a}$ and $\Delta i_{a z}$, denoted by $S_{O P}$ becomes negative for forward fault. In a similar way, $S_{O P}$ becomes positive for reverse fault. Thus by comparing the polarities of $\Delta v_{a}$ and $\Delta i_{a z}$ waveforms direction information of the fault can be acquired.

Presence of multiple frequency components during power swing modulates the voltage and current signals and thereby changes the relative polarity of $\Delta v_{a}$ and $\Delta i_{a z}$. Thus the performance of such directional relaying may be affected. Such a case is demonstrated below for an AG fault created in line 26-28 at a distance of $0.4 \mathrm{pu}$ with $R_{F}=5 \Omega$ during a power swing situation. Fig. 16 (a) and (b) show the phase-A voltage and current waveforms for the situation, whereas the single frequency components extracted from those signals are shown in Fig. 16 (c) and (d).

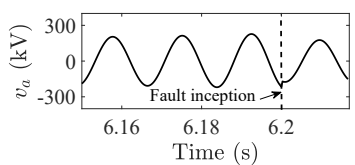

(a)

(c)

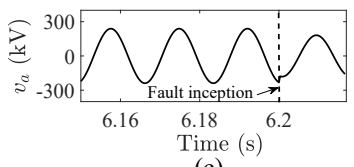

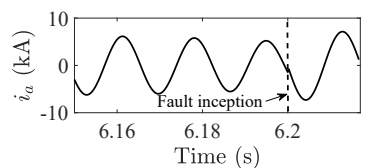

(b)

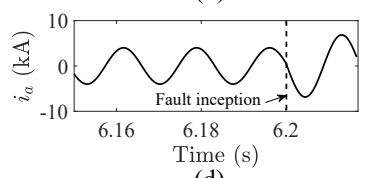

(d)
Fig. 16. (a) Phase-A voltage and (b) phase-A current measured by the relay. (c) single frequency component of measured voltage signal and (d) single frequency component of measured current signal

Results in Fig. 17 (a) and (c) demonstrate the performance of time-domain superimposed component based directional relaying. $S_{O P}$ with positive value, as shown in Fig. 17 (c) indicates that the relay malfunctions in such a situation. On the other hand, $\Delta v_{a}$ and $\Delta i_{a z}$ computed from the single frequency signals of Fig. 16 (c) and (d) are shown in Fig. 17 (b). Result in Fig. 17 (d) demonstrates that the relay employing proposed approach calculates $S_{O P}$ as negative. Thus the fault direction is identified correctly. This confirms the effectiveness of the proposed approach compared to conventional technique even for time domain based protection applications.

\section{Performance of the proposed method for calculating fault location during power swing}

Accurate fault location is required to expedite repair and restoration of lines and avoid lengthy and expensive patrols. Takagi method is one of the well-known approaches used for the purpose [38]. With this approach, the per-unit fault distance for an AG fault is determined using (18) [38].

$$
x=\frac{\operatorname{Im}\left(V_{a} \cdot \Delta I_{1}^{*}\right)}{\operatorname{Im}\left(Z_{1 L} \cdot\left(I_{a}+K_{0} I_{0}\right) \cdot \Delta I_{1}^{*}\right)}
$$




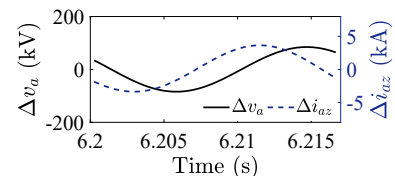

(a)

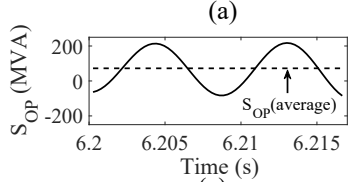

(c)

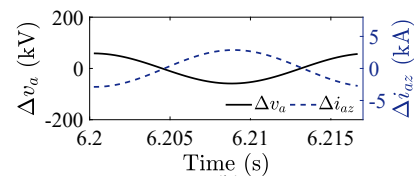

(b)

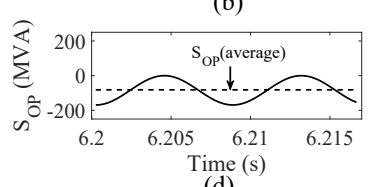

(d)
Fig. 17. Phase-A voltage and replica current waveform obtained from (a) measured signals and (b) single frequency component extraction. Performance of superimposed component based time-domain directional relaying using (c) conventional and (d) proposed approaches.

Where $V_{a}$ and $\left(I_{a}+K_{0} I_{0}\right)$ are the operating voltage and current for the relay. $Z_{1 L}$ is the positive sequence impedance of the protected line. $K_{0}$ represents the zero sequence compensation factor. Expanding the complex variables used in (18), the relation can be rewritten as in (19).

$$
x=\frac{\left|V_{a}\right| \sin \left(\theta_{v}-\alpha\right)}{\left|Z_{1 L}\right|\left|I_{a}+K_{0} I_{0}\right| \sin \left(\theta_{1 L}+\theta_{i}-\alpha\right)}
$$

Where $\theta_{v}, \theta_{i}, \theta_{1 L}$ and $\alpha$ are the angles associated with $V_{a},\left(I_{a}+K_{0} I_{0}\right), Z_{1} L$ and $\Delta I_{1}$ respectively. The incorrect computation of $\Delta I_{1}$ during power swing results in $\alpha$ to be erroneous and thereby affecting the calculation of fault distance using (19). In addition, frequency modulation during power swing changes $Z_{1 L}$ and $K_{0}$ by influencing the line reactance. The incorrect phasor computation in the presence of multi-frequency signals during power swing may also amplify the fault location error. The proposed method overcomes such sources of errors by correct computation of superimposed components using single frequency data extracted from the swing signal, and frequency based adjustment in the line impedances.

A fault location case is demonstrated for an AG fault created in line 26-28 at a distance of $40 \mathrm{~km}$ from bus 28 with $R_{F}=10 \Omega$ during a power swing situation. Fig. 18 shows the differences observed in $\left|V_{a}\right|,\left|I_{a}+K_{0} I_{0}\right|, \theta_{v}, \theta_{i}$ and $\alpha$, as computed by the relay at bus 28 with conventional and proposed approaches. It is observed that $Z_{1 L}$ and $K_{0}$ computed using fundamental frequency $(60 \mathrm{~Hz})$ are $28.74 \angle 86^{0} \Omega$ and $1.94 \angle-4.89^{\circ}$ respectively. On the other hand, those parameters are computed as $27.79 \angle 85.87^{\circ} \Omega$ and $1.94 \angle-5.06^{0}$, when the frequency reference is changed to $58 \mathrm{~Hz}$ (one of the frequencies extracted from the swing signal). Fig. 19 shows the performance of the relay in calculating the fault distance using conventional and proposed approaches. Result shows that the fault distance calculated using the conventional approach is $81.17 \mathrm{~km}$ (after 1-cycle following fault inception), whereas the proposed method calculates the distance as $40.07 \mathrm{~km}$ which is close to the actual fault distance of $40 \mathrm{~km}$. The possibility of any inherent error in computing fault location using Takagi method due to system non-homogeneity is eliminated here by modifying the system impedances suitably. Performance of Takagi method for faults at different locations and fault resistances are provided in Table III using both conventional and proposed approaches. This demonstrates the superiority of the proposed method in improving the performance of such an approach in determining the fault location.

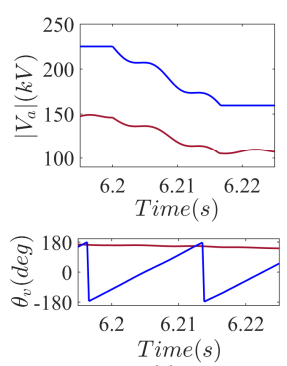

(a)

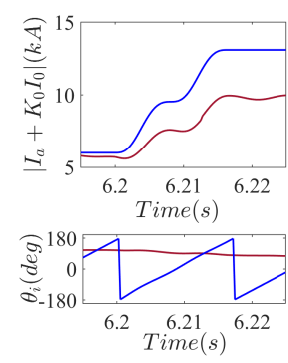

(b)

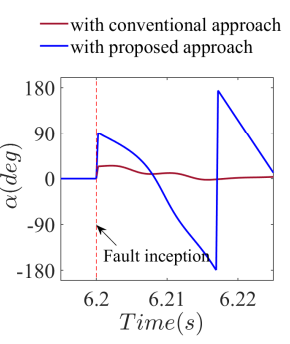

(c)
Fig. 18. Differences observed in (a) $V_{a}$, (b) $\left(I_{a}+K_{0} I_{0}\right)$ and (c) $\alpha$ with conventional and proposed approaches.

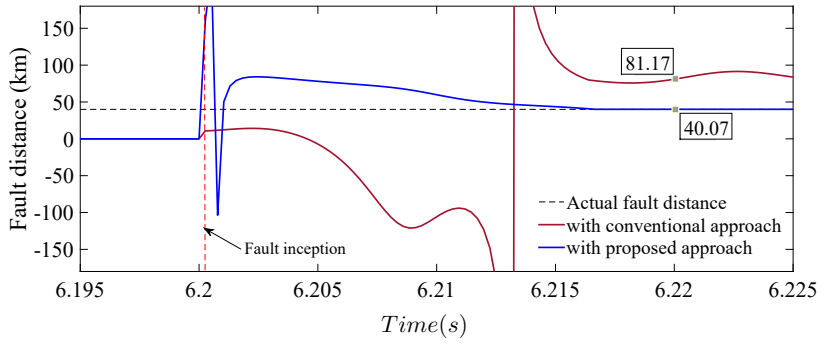

Fig. 19. Performance of proposed method in acquiring correct fault distance.

TABLE III

PERFORMANCE OF TAKAGI METHOD WITH COMPARATIVE ASSESSMENT

\begin{tabular}{|c|c|c|c|c|c|c|}
\hline \multirow{2}{*}{$\begin{array}{l}x_{a c t} \\
(p u)\end{array}$} & \multirow{2}{*}{$\begin{array}{c}x_{\text {calc }}(p u) \\
\text { (Applied approach) }\end{array}$} & \multicolumn{5}{|c|}{$R_{F}$} \\
\hline & & $0.5 \Omega$ & $5 \Omega$ & $15 \Omega$ & $50 \Omega$ & $100 \Omega$ \\
\hline \multirow{2}{*}{0.1} & $x_{\text {calc }}$ (Conventional) & 0.014 & 0.180 & 0.373 & 1.839 & -4.068 \\
\hline & $x_{\text {calc }}($ Proposed $)$ & 0.100 & 0.101 & 0.102 & 0.109 & 0.118 \\
\hline \multirow{2}{*}{0.5} & $x_{\text {calc }}$ (Conventional) & 0.495 & 0.612 & 0.923 & 4.200 & -3.308 \\
\hline & $x_{\text {calc }}($ Proposed $)$ & 0.500 & 0.501 & 0.504 & 0.515 & 0.529 \\
\hline \multirow{2}{*}{0.9} & $x_{\text {calc }}$ (Conventional) & 0.897 & 1.073 & 1.568 & 11.716 & -2.788 \\
\hline & $x_{\text {calc }}($ Proposed $)$ & 0.900 & 0.902 & 0.907 & 0.922 & 0.943 \\
\hline
\end{tabular}

\section{REAL-TIME VALIDATION}

The proposed method is validated using OP4510 (OPALRT) real-time simulator. Fig. 20 shows the real-time simulation experimental setup used for this work. Parameters associated with real-time simulator are provided in Table IV. The 39-bus New England system (of Fig. 1), the proposed superimposed component computation algorithm and the relaying algorithm are modeled in MATLAB integrated with OPAL-RT, which are compiled with RT-LAB to run as an effective platform for developing and testing real-time operation of the proposed method. The fault location technique, available in [38], is tested for an AG fault created in line 26-28 at a distance of $0.9 \mathrm{pu}$ from the relay location at bus 28 with with $R_{F}=10 \Omega$. Using conventional approach the error is found to be $19.22 \%$, whereas it is only $0.28 \%$ when the superimposed component is computed using proposed approach and applied to the fault location algorithm. This demonstrates that the compatibility of the proposed method for real-time application. 


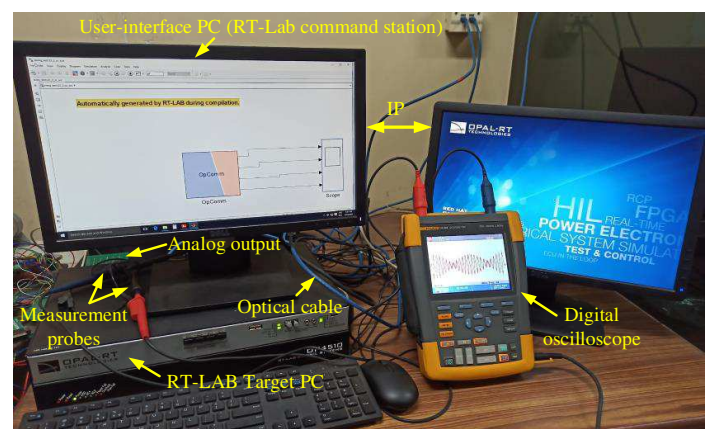

Fig. 20. Real-time simulation (OPAL-RT) experimental setup.

TABLE IV

PARAMETERS ASSOCIATED WITH OPAL-RT SIMULATOR

\begin{tabular}{l|l}
\hline \multicolumn{1}{c|}{ Parameters } & \multicolumn{1}{c}{ Description } \\
\hline Time step & $100 \mu \mathrm{s}$ \\
\hline Operating system & Linux based Redhat OS \\
\hline Simulator & OP4510 (RCP/ HIL vitex7 FPGA processor) \\
\hline
\end{tabular}

\section{CONCLUSION}

Power swing results in modulation in amplitude, phase, and frequency of voltage and current signals. This creates issues in obtaining accurate non-fault component for fault during power swing and resulting in superimposed components be erroneous. The protection schemes employing such incorrect superimposed component may be affected in deriving correct decisions. This work proposes a method to compute superimposed components correctly for fault during power swing and gives a scope to enhance the performance of associated protection schemes for such a situation. The method extrapolates the pre-fault signal to acquire the correct non-fault component during fault for computing superimposed quantity correctly for the situation. The proposed method demodulates the swing signal and the associated protection decision can be obtained by using any single frequency component as extracted from the superimposed signal. The method is adaptive to variation in swing frequency. The accuracy of non-fault component estimation method is tested for different swing conditions with variation in swing frequency and its rate, power angle variation, and in the presence of measurement noise and harmonics. The performance is also evaluated using power swing field data and the compatibility of the proposed method is checked with real-time simulator. The improved performance of different protection schemes are observed for faults during power swing with changes in fault type, fault location, fault resistance, swing frequency, and fault inception angle. The proposed method is applied for both time and phasor domain protection applications. Comparative assessment reveals the superiority of the proposed method.

\section{REFERENCES}

[1] M. McDonald, D. Tziouvaras, A. Apostolov et al., "Power swing and out-of-step considerations on transmission lines," IEEE PSRC WG D6, vol. 6, p. 2005, 2005.

[2] N. Fischer, G. Benmouyal, D. Hou, D. Tziouvaras, J. Byrne-Finley, and B. Smyth, "Tutorial on power swing blocking and out-of-step tripping," in Proc. 39th Аnnu. Western Protective Relay Conf., 2012, pp. 1-20.
[3] D. Novosel, G. Bartok, G. Henneberg, P. Mysore, D. Tziouvaras, and S. Ward, "IEEE PSRC report on performance of relaying during widearea stressed conditions," IEEE Trans. Power Del., vol. 25, no. 1, pp. $3-16,2010$.

[4] M. Abdi-Khorsand and V. Vittal, "Modeling protection systems in timedomain simulations: A new method to detect mis-operating relays for unstable power swings," IEEE Trans. Power Sys., vol. 32, no. 4, pp. 2790-2798, 2017.

[5] B. Patel, P. Bera, and S. H. Nee Dey, "A novel method to distinguish internal and external faults during power swing," IEEE Trans. Power Del., vol. 36, no. 5, pp. 2595-2605, 2021.

[6] S. Das, B. K. Panigrahi, and P. K. Jaiswal, "Qualitative assessment of power swing for enhancing security of distance relay in a TCSCcompensated line," IEEE Trans. Power Del., vol. 36, no. 1, pp. 223-234, 2021.

[7] "Protection system response to power swings," System Protection and Control Subcommittee, NERC, Atlanta, GA, Tech. Rep., August 2013.

[8] "Report of the enquiry committee on grid disturbance in Northern Region on 30th july 2012 and in Northern, Eastern and North-Eastern region on 31st july 2012," Powergrid, New Delhi, India, Tech. Rep., August 2012.

[9] "Effect of solar PV on transient stability of the new zealand power system," Transpower New Zealand Limited, Tech. Rep., December 2017.

[10] A. Haddadi, I. Kocar, U. Karaagac, H. Gras, and E. Farantatos, "Impact of wind generation on power swing protection," IEEE Trans. Power Del., vol. 34, no. 3, pp. 1118-1128, 2019.

[11] S. McGuinness, M. Patel, and A. Kelly, "Performance of protection relays during stable and unstable power swings," The Journal of Engineering, vol. 2018, no. 15, pp. 1125-1129, 2018.

[12] D. Kang and R. Gokaraju, "A new method for blocking third-zone distance relays during stable power swings," IEEE Trans. Power Del., vol. 31, no. 4, pp. 1836-1843, 2016.

[13] B. Patel and P. Bera, "Detection of power swing and fault during power swing using lissajous figure," IEEE Trans. Power Del., vol. 33, no. 6 , pp. 3019-3027, 2018.

[14] S. A. Lavand and S. A. Soman, "Predictive analytic to supervise zone 1 of distance relay using synchrophasors," IEEE Trans. Power Del., vol. 31, no. 4, pp. 1844-1854, 2016.

[15] M. K. Jena and B. K. Panigrahi, "Transient potential power based supervisory zone-1 operation during unstable power swing," IEEE Syst. J., vol. 13, no. 2, pp. 1823-1830, 2019.

[16] W. Li, T. Bi, and Q. Yang, "Study on sequence component based fault phase selector during power swings," in 2010 5th International Conference on Critical Infrastructure (CRIS), 2010, pp. 1-5.

[17] Li Zou, Qingchun Zhao, Xiangning Lin, and Pei Liu, "Improved phase selector for unbalanced faults during power swings using morphological technique," IEEE Trans. Power Del., vol. 21, no. 4, pp. 1847-1855, 2006.

[18] P. Jena and A. K. Pradhan, "Directional relaying during power swing and single-pole tripping," in International Conference on Power Systems, 2009, pp. 1-6.

[19] Y. Xue, B. Kasztenny, D. Taylor, and Y. Xia, "Series compensation, power swings, and inverter-based sources and their impact on line current differential protection," in 66th Annual Conference for Protective Relay Engineers, 2013, pp. 80-91.

[20] P. K. Nayak, A. K. Pradhan, and P. Bajpai, "A three-terminal line protection scheme immune to power swing," IEEE Trans. Power Del., vol. 31, no. 3, pp. 999-1006, 2016.

[21] E. O. Schweitzer, B. Kasztenny, A. Guzmn, V. Skendzic, and M. V. Mynam, "Speed of line protection - can we break free of phasor limitations?" in 68th Annual Conference for Protective Relay Engineers, 2015, pp. 448-461.

[22] E. O. Schweitzer, B. Kasztenny, and M. V. Mynam, "Performance of time-domain line protection elements on real-world faults," in 69th Annual Conference for Protective Relay Engineers (CPRE), 2016, pp. $1-17$.

[23] G. Benmouyal and J. Roberts, "Superimposed quantities: their true nature and application in relays," in 26th Annual Western Protective Relay Conference, 1999, pp. 1-18.

[24] P. Horton and S. Swain, "Using superimposed principles (delta) in protection techniques in an increasingly challenging power network," in 70th Annual Conference for Protective Relay Engineers (CPRE), 2017, pp. 1-12.

[25] Qing-Qiang Xu, J. Suonan, and Yao-Zhong Ge, "Real-time measurement of mean frequency in two-machine system during power swings," IEEE Trans. Power Del., vol. 19, no. 3, pp. 1018-1023, 2004. 
[26] J. Khodaparast and M. Khederzadeh, "Three-phase fault detection during power swing by transient monitor," IEEE Trans. Power Syst., vol. 30 no. 5, pp. 2558-2565, 2015.

[27] S. Paladhi and A. K. Pradhan, "Adaptive distance protection for lines connecting converter-interfaced renewable plants," IEEE J. Emerg. Selec. Topics Power Electron., vol. 9, no. 6, pp. 7088-7098, 2021.

[28] S. Paladhi and A. K. Pradhan, "Adaptive fault type classification for transmission network connecting converter-interfaced renewable plants,' IEEE Syst. J., vol. 15, no. 3, pp. 4025-4036, 2021.

[29] A. Apostolov, D. Tholomier, and S. Richards, "Superimposed components based sub-cycle protection of transmission lines," in IEEE PES Power Systems Conference and Exposition, 2004, pp. 592-597 vol.1.

[30] M. Biswal, B. B. Pati, and A. K. Pradhan, "Directional relaying for double circuit line with series compensation," IET Gen., Trans. Distr. vol. 7, no. 4, pp. 405-413, 2013.

[31] D. Yuan, X. Dong, S. Chen, Z. Q. Bo, B. R. J. Caunce, and A. Klimek, "An new directional comparison scheme for distribution line protection," in Proc. 41st International Universities Power Engineering Conference, vol. 3, 2006, pp. 881-885.

[32] B. Mahamedi and J. E. Fletcher, "Setting-free method for detection of asymmetrical faults during power swings," Electric Power System Research, vol. 181, 2020.

[33] B. Kasztenny, A. Guzmán, N. Fischer, M. V. Mynam, and D. Taylor, "Practical setting considerations for protective relays that use incremental quantities and traveling waves," in 43rd Annual Western Protective Relay Conference, Washington, USA, 2016, pp. 1-25.

[34] M. S. Sachdev and M. A. Baribeau, "A new algorithm for digital impedance relays," IEEE Trans. Power Appar. Syst., vol. PAS-98, no. 6, pp. 2232-2240, 1979.

[35] S. Das and T. Sidhu, "A simple synchrophasor estimation algorithm considering ieee standard c37.118.1-2011 and protection requirements," IEEE Trans. Instr. Meas., vol. 62, no. 10, pp. 2704-2715, 2013.

[36] B. Kasztenny, B. Campbell, and J. Mazereeuw, "Phase selection for single-pole tripping weak infeed conditions and cross-country faults," GE Power Management, Madrid, Spain, Tech. Rep. GER-3997, Oct. 2000.

[37] GE Mulltilin, "D90 plus line distance protection system," 2012, instruction manual.
[38] K. Zimmerman and D. Costello, "Impedance-based fault location experience," in 58th Annual Conference for Protective Relay Engineers, 2005, pp. 211-226.

Subhadeep Paladhi received $\mathrm{PhD}$ degree in Electrical Engineering from Indian Institute of Technology, Kharagpur, India in 2021. He is currently working as a research associate in the Department of Electronic and Electrical Engineering, University of Strathclyde, Glasgow, UK. His research interest is power system protection.

Ashok Kumar Pradhan received the Ph.D. degree in Electrical Engineering from Sambalpur University, Sambalpur, India, in 2001. He has been with the Department of Electrical Engineering, Indian Institute of Technology, Kharagpur, India, since 2002, where he is a Professor. His research interests include power system relaying and monitoring.

Prof. Pradhan is a Fellow of the Indian National Academy of Engineering, India, and a Fellow of National Academy of Sciences, India.

J. Ganeswara Rao received M. Tech and Ph.D. in Electrical Engineering from Indian institute of Technology, Kharagpur, India in 2011 and 2016 respectively. Presently he is with Central Electricity Authority, India. His research area is power system protection. 